\title{
Titanium effect on the microstructure and properties of the layer deposited by Fe-C-Si-Mn- Cr-Mo-Ni flux cored wire on the parts working in severe wear conditions
}

\author{
Nikolay Kozyrev*, Alexey Mikhno, Aleksandr Usoltsev, Natalya Kibko, and Olga Kozyreva \\ Siberian State Industrial University, Novokuznetsk, 654000, Russia

\begin{abstract}
The study results of the structure and properties of the layer deposited with flux-cored wires of the Fe-C-Si-Mn-Cr-Mo-Ni system additionally alloyed with titanium are presented. Surfacing with the investigated flux-cored wires was carried out on a substrate made of 09G2S steel using silicomanganese slag flux.
\end{abstract}

\section{Introduction}

Mechanisms of mining equipment subjected to abrasive and impact wear during operation fail prematurely. The wear of the working surfaces makes it necessary to restore them. Therefore, the development of materials that significantly increase the wear resistance of such parts, and the use of technology for their recovery, is an important task. The most promising is the deposition with flux-cored wire on the wearing surfaces of parts.

The quality of the deposited (restored) layer during the repair of parts operating in the conditions of intense wear depends to a greater extent on the choice of welding consumables and their technological properties. The composition of these consumables completely determines the composition of liquid slag and arc atmosphere, the chemical composition of the deposited layer, which, in its turn, influences the structure, mechanical properties of the restored part [1-6].

In this work, we studied the effect of titanium powder (PTS grade), introduced into the composition of flux-cored wire, on the microstructure, hardness and wear resistance of the layer deposited by it. In laboratory conditions, samples of flux-cored wires were made using appropriate powdered materials. In the course of the study, the chemical composition of the deposited metal was determined, metallographic analysis was carried out, and the mechanical properties were described.

As a result of metallography, the following was established: the size of the former austenite grain, the martensite dispersion in the structure of the deposited metal, the level of its contamination with non-metallic inclusions. It was found that an increase in the titanium content from 0.008 to $(0.013-0.035) \%$ with a simultaneous change in the chemical composition contributes to the grinding of martensite needles in the structure of the

\footnotetext{
*Corresponding author: kozyrev_na@mtsp.sibsiu.ru
} 
deposited layer and to the increase in hardness and wear resistance. A change in the degree of alloying of flux-cored wire of the Fe-C-Si-Mn-Cr-Mo-Ni system affects the level of contamination with non-metallic inclusions of the layer deposited by it.

\section{Materials and research methods}

Wires were made with a calculated (without taking into losses) content of titanium powder in order to study the effect of introducing titanium powder into flux-cored wires on the microstructure, hardness and wear resistance during submerged arc welding with manganese-containing flux.

For the manufacture of flux-cored wires, powder materials were used: carbon-fluorinecontaining material (gas cleaning dust from aluminum production), iron powder PZhV-1 according to Russian national standards GOST 9849-86, chromium powder PKh-1S according to technical requirements TU 14-1-1474-75, manganese powder MR-0 according to GOST 6008-82, silicon powder KR-1 according to GOST 2169-69, molybdenum powder MPCh according to TU 48-19-69-80 nickel powder PNK-1L5 according to GOST 9722-97, titanium powder PTS according to TU 14-22-57- 92.

Surfacing of the samples was carried out on a substrate made of steel grade 09G2S using a welding flux made of silicomanganese slag, developed at Siberian State Industrial University [7-9], with a chemical composition, mass: in this work, $0.52 \mathrm{FeO} ; 16.15 \mathrm{MnO}$; $29.13 \mathrm{CaO} ; 42.40 \mathrm{SiO}_{2} ; 6.80 \mathrm{Al}_{2} \mathrm{O}_{3} ; 1.39 \mathrm{MgO} ; 0.18 \mathrm{Na} 2 \mathrm{O} ; 0.59 \mathrm{~K}_{2} \mathrm{O} ; 0.23 \mathrm{~S} ; 0.022 \mathrm{P}$; $0.004 \mathrm{ZnO} ; 0.024 \mathrm{C} ; 0.32 \mathrm{~F} ; 0.17 \mathrm{TiO}_{2} ; 0.033 \mathrm{Cr}_{2} \mathrm{O} 3$.

The deposited layer chemical composition of the samples under study was examined by the atomic emission method on the DFS-71 spectrometer. The chemical composition analysis of the slag crusts was carried out by the X-ray fluorescence method on the XRF1800 spectrometer.

The study of the microstructure and the degree of contamination with non-metallic inclusions of the layer deposited with a flux-cored wire of the Fe-C-Cr-Mn-Si-Mo-Ni-Ti system was carried out using metallographic microscopes METAM PB-34 and OLYMPUS GX-51 in a bright field in the range of magnifications from $\times 100$ to $\times 1000$ using the NEXSYS ImageExpert Sample 2 and Siams Photolab 700 software for metallography, respectively. NEXSYS ImageExpert Sample 2 software is designed for high-quality image analysis by visual comparison of the thin section viewing fields with reference scales or dimensional grids. The ability of the program to store in the computer all the reference scales needed for work makes it possible to observe simultaneously the investigated structure and the standard on the monitor screen at equal magnification, to carry out a comparative analysis of the microstructure and, on its basis, generate a report including statistical parameters, distribution histograms and the original image. The program makes it possible to accumulate analysis results, both for a number of viewing fields in accordance with a single scale, and for a number of scales within one standard. The program implements a linear meter unit, which allows single measurements of the linear dimensions of structural components to be performed, both in the captured images of real structures and in the images of the reference scales presented in the program. The program quickly estimates the size of structural elements in the image by overlaying scale grids: measured segment, crosshair, mesh.

The study of the degree of deposited layer contamination with non-metallic inclusions was carried out using the software of the METAM RV-34 microscope in accordance with GOST 1778-70. The polished surface of longitudinal sections was studied at a magnification of $\times 100$ by comparison with the reference scales included in the program. The size of the primary austenite grain and the characteristics of martensite in the structure of the layer deposited with flux-cored wire were investigated after etching polished sections 
in a $4 \%$ solution of $\mathrm{HNO}_{3}$ using a METAM PB-34 metallographic microscope according to GOST 5639-82 at magnification of $\times 100$ and according to GOST 8233-56 at magnification $\times 1000$ by comparison with reference scales. The grain size was found using a reference scale, included in the application program for determining the grain size in steels, by visual comparison of the structure under study and the reference. At the same time, the analyzed structure was assigned an appropriate point and a report was generated on a series of viewing fields. The size of the martensite needles was determined using a linear meter unit.

The hardness of the layer deposited with flux-cored wire was measured by the Brinell and Rockwell methods in accordance with the requirements of GOST 9012-59 and GOST 9013-59.

The wear resistance of the deposited layer of the test samples was determined by carrying out wear tests on a 2070 SMT-1 machine.

\section{Results and discussion}

The chemical composition of the deposited layer and slag crusts, determined by X-ray fluorescence and atomic emission methods, is presented in Tables 1 and 2.

Table 1. Chemical composition of the deposited layer.

\begin{tabular}{|c|c|c|c|c|c|c|c|c|c|c|c|c|c|}
\hline \multirow{2}{*}{$\begin{array}{c}\text { Sample } \\
\text { No. }\end{array}$} & $\mathbf{C}$ & $\mathbf{S i}$ & $\mathbf{M n}$ & $\mathbf{C r}$ & $\mathbf{N i}$ & $\mathbf{C u}$ & $\mathbf{T i}$ & $\mathbf{V}$ & $\mathbf{M o}$ & $\mathbf{A l}$ & $\mathbf{N b}$ & $\mathbf{S}$ & $\mathbf{P}$ \\
\hline S1 & 0.24 & 1.36 & 1.44 & 4.75 & 0.34 & 0.05 & 0.008 & 0.004 & 0.62 & 0.012 & 0.003 & 0.050 & 0.019 \\
\hline S2 & 0.24 & 1.72 & 1.19 & 5.97 & 0.37 & 0.06 & 0.013 & 0.005 & 0.85 & 0.013 & 0.003 & 0.055 & 0.026 \\
\hline S3 & 0.27 & 2.51 & 1.61 & 4.64 & 0.27 & 0.06 & 0.019 & 0.004 & 0.67 & 0.043 & 0.004 & 0.034 & 0.019 \\
\hline S4 & 0.27 & 2.66 & 1.68 & 4.63 & 0.27 & 0.06 & 0.035 & 0.004 & 0.64 & 0.045 & 0.004 & 0.044 & 0.010 \\
\hline
\end{tabular}

Table 2. Chemical composition of slag crusts.

\begin{tabular}{|c|c|c|c|c|c|c|c|c|c|c|c|c|c|c|c|c|c|c|}
\hline$\dot{8}$ & & & & & & & & ass fra & action & of ele & ments, & & & & & & & \\
\hline 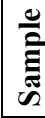 & : & $\stackrel{\stackrel{O}{E}}{\sum}$ & ల్ల & $\stackrel{0}{\infty}$ & 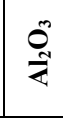 & $\sum_{\sum}^{\infty}$ & $\begin{array}{l}\text { O } \\
\text { ż }\end{array}$ & $\begin{array}{l}0 \\
\text { ON }\end{array}$ & $n$ & $a$ & $\stackrel{\text { O }}{\text { N }}$ & u & 5 & O్ & $\stackrel{0}{0}$ & $\stackrel{0}{D}$ & $\begin{array}{l}\text { రే } \\
\tilde{U}\end{array}$ & $0^{\infty}$ \\
\hline S1 & 1.84 & 6.27 & 35.49 & 43.53 & 7.51 & 1.32 & 0.32 & 0.59 & 0.21 & 0.021 & 0.005 & 0.041 & 0.33 & 0.88 & 0.14 & 0.27 & $|0.46|$ & 0.001 \\
\hline S2 & 1.98 & 6.23 & 36.48 & 41.58 & 7.52 & 1.15 & $0.27 \mid$ & 0.58 & 0.21 & 0.021 & 0.007 & 0.070 & 0.29 & 0.97 & 0.15 & 0.29 & 0.35 & 0.28 \\
\hline S3 & 2.34 & 6.24 & 34.52 & 41.45 & 7.75 & 1.31 & 0.40 & 0.60 & 0.24 & 0.021 & 0.003 & 0.057 & 0.66 & 0.85 & 0.16 & 0.29 & 0.76 & 0.53 \\
\hline S4 & 1.65 & 6.23 & 35.87 & 42.26 & 7.19 & 1.11 & 0.37 & 0.59 & 0.21 & 0.021 & 0.006 & 0.062 & 0.37 & 0.91 & 0.14 & 0.28 & 0.77 & 0.015 \\
\hline
\end{tabular}

As an examination result of the nature of non-metallic inclusions of the deposited layer with flux-cored wire of the Fe-C-Cr-Mn-Si-Mo-Ni-Ti system, contamination with oxide non-metallic inclusions, in particular non-deformable silicates and point oxides, was revealed (Table 3, Fig. 1).

It was established that a change in the chemical composition of the investigated fluxcored wire affects the level of contamination with non-metallic inclusions of the layer deposited by it. 
Table 3. Characteristics of non-metallic inclusions of the test samples.

\begin{tabular}{|c|c|c|c|}
\hline \multirow{2}{*}{ Sample No. } & \multicolumn{3}{|c|}{ Contamination with non-metallic inclusions, point } \\
\cline { 2 - 4 } & Point oxides & Non-deformable silicates & Point nitrides \\
\hline S1 & $3 \mathrm{a}$ & $3 \mathrm{a}$ & - \\
\hline S2 & $4 \mathrm{a}$ & $2 \mathrm{a}$ & - \\
\hline S3 & $4 \mathrm{a}$ & $2 \mathrm{~b}$ & $1 \mathrm{a}$ \\
\hline S4 & $4 \mathrm{a}$ & $5 \mathrm{~b}$ & $2 \mathrm{a}$ \\
\hline
\end{tabular}

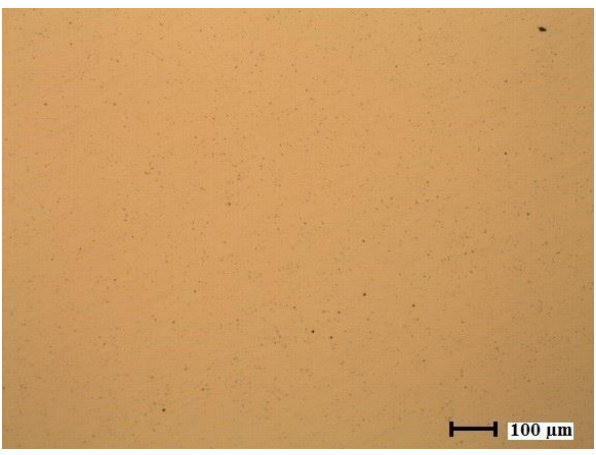

a

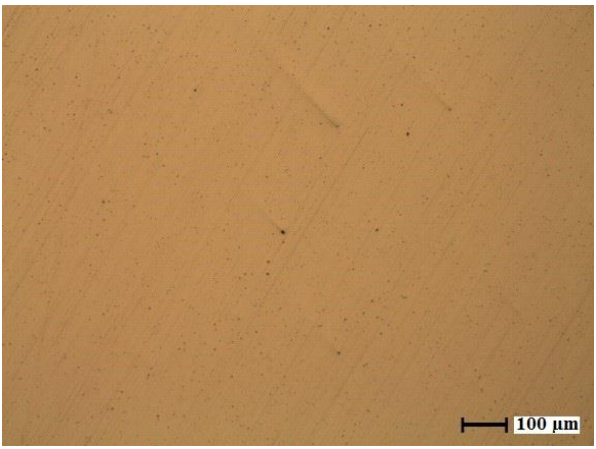

$\mathrm{c}$

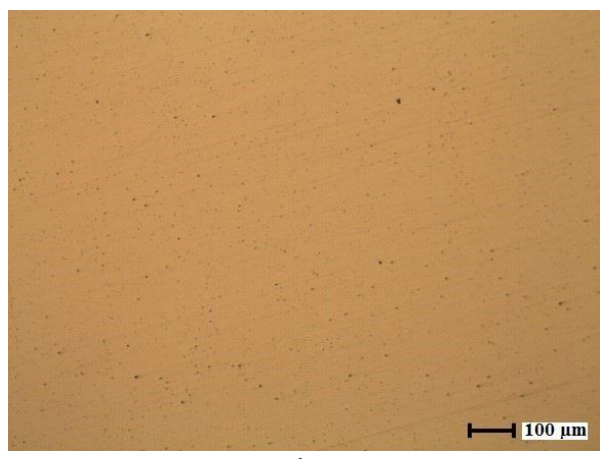

b

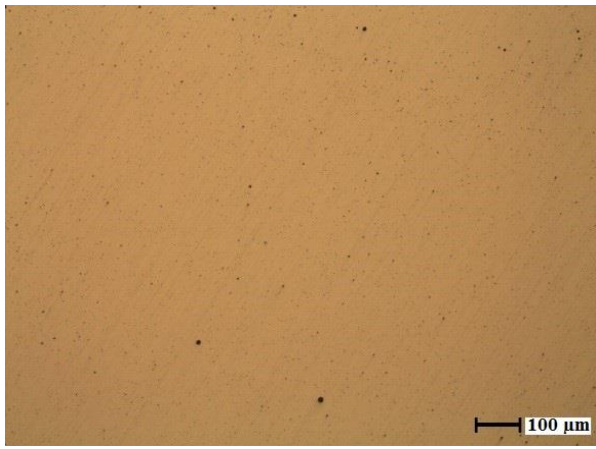

d

a - sample S1, b - sample S2, c - sample S3, d - sample S4

Fig. 1. Non-metallic inclusions of the layer deposited with flux-cored wire of the Fe-C-Cr-Mn-Si-MoNi-Ti system.

The smallest contamination of the deposited layer with non-metallic inclusions is observed with a titanium content of $0.008 \%$ and $0.013 \%$ in its composition. With an increase in the titanium content to $0.019 \%$ and $0.035 \%$ in the deposited layer, the presence of point nitrides was established in it, except for non-deformable silicates and point oxides. The microstructure of the deposited layers is uniform, has mainly a dendritic structure and is a fine-acicular and medium-acicular martensite (points 3, 4 and 5) with a needle size (28) $\mu \mathrm{m}$ in the former austenite grains, along the boundaries of which there are thin layers of $\delta$-ferrite, and a small amount of residual austenite in the form of separate areas (Fig. 2, Table 4). In the structure of the deposited layer with a titanium content of $0.008 \%$, the smallest amount of $\delta$-ferrite is observed. The size of the former austenite grain on the grain scale corresponds to No. 5. In the peripheral areas of the deposited layers structure under investigation, there is a finer grained structure (the size of the former austenite grain corresponds to No. 6) in addition to the deposited layer with the lowest titanium content. 


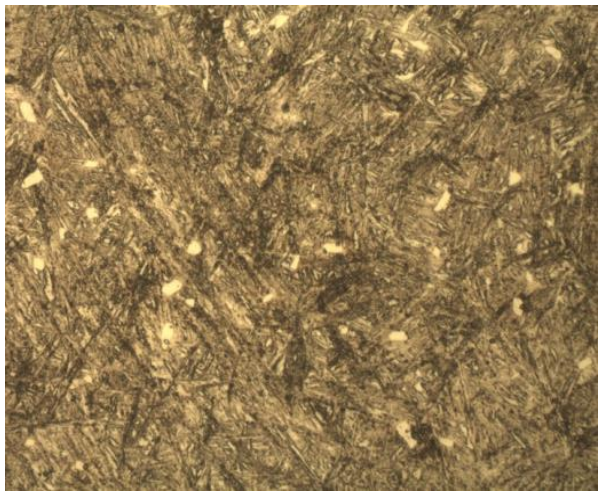

a

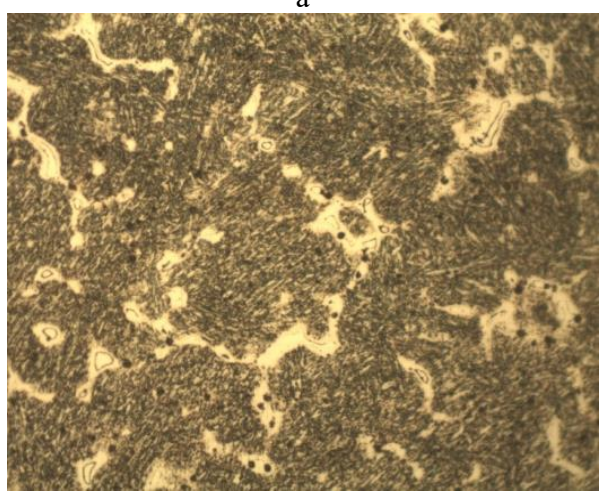

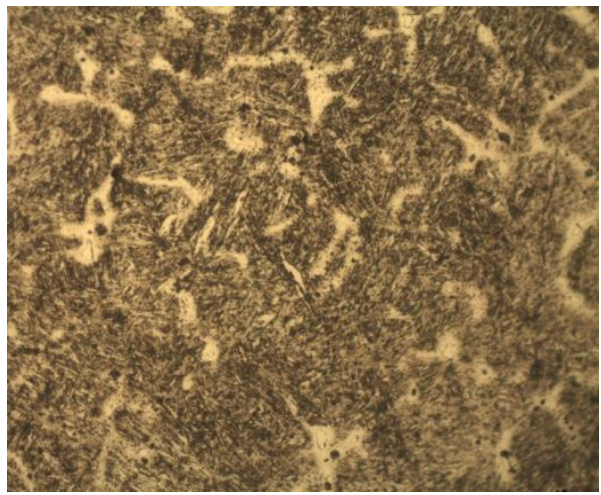

b

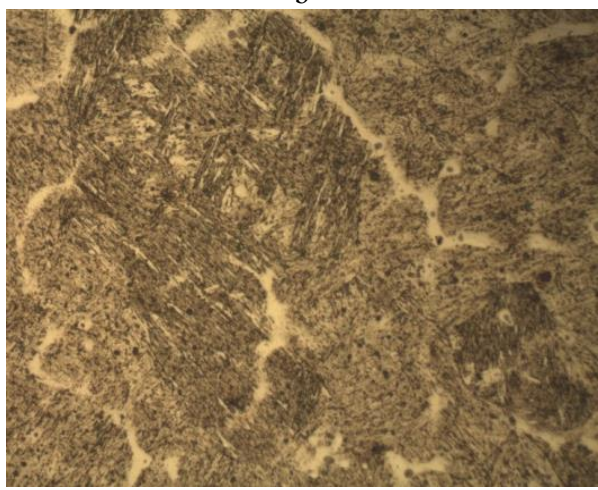

d

a - sample $\mathrm{S} 1, \mathrm{~b}$ - sample S2, c - sample S3, d - sample S4

Fig. 2. Microstructure of layers deposited with flux-cored wire of the Fe-C-Cr-Mn-Si-Mo-Ni-Ti system, $\times 500$.

It was found that an increase in the titanium content from $0.008 \%$ to $(0.013-0.035) \%$ with a simultaneous change in the content of other elements in the deposited layer contributes to a decrease in the size of the martensite needles without affecting the size of the primary austenite grain. Martensite ranges from medium-acicular to fine-acicular. The smallest martensite needles are observed in the structure of deposited layers with titanium content of $0.013 \%$ and $0.019 \%$. An increase in the titanium content in the deposited layer up to $0.035 \%$ ensures the enlargement of the martensite needles (Fig. 2, Table 4).

Table 4. Structure characteristics of the studied samples.

\begin{tabular}{|c|c|c|c|}
\hline Sample No. & Grain No. & Martensite point & Size of martensite needles, $\boldsymbol{\mu m}$ \\
\hline C1 & 5 & 5 & $4-8$ \\
\hline C2 & 5 & 3 & $2-4$ \\
\hline C3 & 5 & 3 & $2-4$ \\
\hline C4 & 5 & 4 & $2-6$ \\
\hline
\end{tabular}

It is shown that changes in the microstructure with varying the chemical composition of the deposited layer are accompanied by the change in its properties.

An increase in the titanium content contributes to an increase in the values of wear resistance and hardness measured by the Brinell and Rockwell methods (Table 5). The best 
results are observed with the highest titanium content in the deposited layer $(0.035 \%)$. However, to a lesser extent it provides for the refinement of martensite in the structure of the deposited layer compared with $0.013 \%$ and $0.019 \%$ titanium.

Table 5. Wear rate and hardness of the test samples.

\begin{tabular}{|c|c|c|c|c|c|}
\hline $\begin{array}{c}\text { Sample } \\
\text { No. }\end{array}$ & $\begin{array}{c}\text { Hardness limits } \\
\text { according to HB }\end{array}$ & $\begin{array}{c}\text { HRC } \\
\text { hardness } \\
\text { limits }\end{array}$ & $\begin{array}{c}\text { Medium } \\
\text { hardness HB }\end{array}$ & $\begin{array}{c}\text { Medium } \\
\text { hardness HRC }\end{array}$ & Wear rate (g/ rev·10-5) \\
\hline S1 & $355-396$ & $33-40$ & 375 & 35 & 7.77 \\
\hline S2 & $410-466$ & $42-47$ & 437 & 44 & 6.59 \\
\hline S3 & $434-581$ & $45-49$ & 506 & 46 & 6.04 \\
\hline S4 & $444-575$ & $44-50$ & 509 & 47 & 5.24 \\
\hline
\end{tabular}

\section{Conclusions}

1. An increase in the titanium content from $0.008 \%$ to $0.035 \%$ with a simultaneous change in the chemical composition of the investigated flux-cored wire affects the level of contamination with non-metallic inclusions of the layer deposited by it. The smallest contamination with non-metallic inclusions is observed when the titanium content in the deposited layer is $0.008 \%$ and $0.013 \%$. For the deposited layers with a higher titanium content of $0.019 \%$ and $0.035 \%$, the presence of point nitrides (except for oxide nonmetallic inclusions) was established.

2. An increase in the titanium content with a simultaneous change in the content of other elements in the composition of the deposited layers obtained using multicomponent wires of the Fe-C-Cr-Mn-Si-Mo-Ni-Ti system ensures fining of the martensite needles. Martensite ranges from medium-acicular to fine-acicular. The greatest effect of improving the microstructure of the layers deposited with flux-cored wire is observed when the content of titanium in it is $0.013 \%$ and $0.019 \%$. Their structure is characterized by the size of the former austenite grains corresponding to the grain size scale No. 5, within the boundaries of which there is fine-needle martensite with needles up to $4 \mu \mathrm{m}$ in size.

3. An increase in the titanium content with a simultaneous change in the chemical composition of the investigated flux-cored wire contributes to an increase in hardness (from 375 to $509 \mathrm{HB}$; from 35 to $47 \mathrm{HRC}$ ) and wear resistance of the deposited layers (the degree of wear decreases from 7.77 to $5.24 \mathrm{~g} / \mathrm{rev} \cdot 10^{-5}$ ).

\section{References}

1. D. Yakovlev, et al., Appl. of Flux-cored Wire in Multi-arc Welding (2016)

2. N. Eremin, A.S. Losev, Omsk Scientific Bulletin, 2, 45-47 (2014)

3. P. Tashev, et al, Nanomidif. in the Process of Welding and Surfacing, 1, 16-21 (2017)

4. Q. Wang, X. Li, Weld. International, 89, 133-139 (2010).

5. G. Sokolov, V.I. Lysak, Hardfacing of Wear-resistant Alloys on Press Dies and Tools for Hot Deformation of Steels (2005)

6. A. Antonov, A.A. Artemiev, et al., Izv. Vuzov. Ferrous Metallurgy, 9, 671-672 (2016)

7. N. Kozyrev, R.E. Kryukov, et al, Development of new welding fluxes based on silicomanganese slag, in Proceedings of Conference on Welding and control (2018)

8. N. Kozyrev, R.E. Kryukov, et al, Welding Production, 2, 16-21 (2020)

9. N. Kozyrev, R.E. Kryukov, et al, Ferrous Metallurgy. Bulletin of Scientific, Technical and Economic Information, 6, 55-66 (2018) 